\title{
OCLUSÃO DENTÁRIA EM POPULAÇÕES INDÍGENAS
}

\section{DENTAL OCCLUSION IN INDIGENOUS POPULATION}

\author{
Juliana Nogueira de Souza Campos* \\ Dênis Clay Lopes dos Santos* \\ Daniel Negrete ${ }^{* *+}$ \\ Everton Flaiban ${ }^{* * * *}$ \\ Pablo Natanael Lemos
}

\begin{abstract}
RESUMO
As doenças bucais são grandes problemas de saúde pública em todas as regiões do mundo e o maior peso dessas doenças é vivenciado por grupos populacionais desfavorecidos. Dentro desses problemas, as oclusopatias apresentam elevada prevalência, ficando atrás apenas da cárie dentária e problemas periodontais. Estudos antropológicos apontam para uma menor exigência do sistema mastigatório nas populações de grandes cidades, resultando em dentições com menor função e eficiência mastigatória e um aumento na prevalência e severidade das oclusopatias. Os povos indígenas no Brasil têm passado também, nas últimas décadas, por uma mudança no modo de viver, na alimentação e no perfil epidemiológico. Os estudos sobre as condições de saúde bucal dos povos indígenas se concentram em doenças como a cárie dentária e a doença periodontal. Há poucos relatos de estudos sobre oclusopatias em indígenas, mas isso tem instigado pesquisadores desde 1964. Foi realizada a busca na literatura de artigos que relatassem aspectos sobre oclusão dentária de povos indígenas no Brasil. Nos estudos encontrados, observa-se a diversidade de padrões relacionados à oclusão dentária. A utilização de metodologias distintas dificulta a comparação entre a prevalência dos vários tipos de má oclusão nas diferentes regiões e grupos étnicos. Conclui-se que mais estudos são necessários para conhecer o perfil epidemiológico das populações indígenas no Brasil no que se refere às oclusopatias e deve-se buscar mecanismos para que os estudos utilizem uma mesma metodologia, de forma a facilitar a comparação entre dados de diferentes estudos.
\end{abstract}

Descritores: Saúde bucal • Oclusão dentária • População indígena • Índios sul-americanos

\section{ABSTRACT}

Oral diseases are major public health problems in all regions of the world and the greatest burden of these diseases is experienced by disadvantaged population groups. Within these problems, the occlusopathies present a high prevalence, behind only dental caries and periodontal problems. Anthropological studies report a lower requirement of the masticatory system in the populations of large cities, resulting in dentitions with less function and masticatory efficiency and an increase in the prevalence and severity of the malocclusion. Indigenous population in Brazil, in the last decades, have also gone through changes in their way of living, in the diet and in the epidemiological profile. Studies on the oral health conditions of indigenous peoples focus on diseases such as dental caries and periodontal disease. There are few reports of studies on occlusion in indigenous people, but this has instigated researchers since 1964. A search was made in the literature for articles that reported aspects about dental occlusion of indigenous population in Brazil in the period from 1964 to 2015. In the studies the diversity of dental occlusion-related patterns was observed. The use of different methodologies makes it difficult to compare the prevalence of various types of malocclusion in different regions and ethnic groups. It is concluded that more studies are needed to know the epidemiological profile of the indigenous population in Brazil regarding the malocclusion; and mechanisms should be sought so that the studies use the same methodology, in order to facilitate the comparison of data from different studies.

Descriptors: Oral health • Dental occlusion • Indigenous population • Indians, South American

* Especialista em Saúde Indígena - Universidade Federal de São Paulo. Especialista em Ortodontia - Universidade Cruzeiro do Sul. e-mail: ju.nog.souza@ gmail.com

** Coordenador do Curso de Odontologia da Universidade Cruzeiro do sul. Professor Titular da disciplina de Ortodontia e Ortopedia Funcional da Universidade Cruzeiro do Sul. Coordenador da especialização em Ortodontia da Universidade Cruzeiro do Sul. Coordenador da especialização em implantodontia da Universidade Cruzeiro do Sul. Doutorado e Mestrado-FCM-UNICAMP. PhD em Ortodontia e Ortopedia Facial. PhD em Implantodontia e-mail: dr.denis@globomail.com

*** Mestre em Ortodontia pela Unicid. Professor assistente do Curso de Especialização em Ortodontia da Universidade Cruzeiro do Sul. Professor assistente do Curso de Especialização em Ortodontia da Universidade de Uberaba - UNIUBE e-mail: daninegrete75@gmail.com

**** Mestre em Ortodontia - UNICID. Especialista em Ortodontia e Ortopedia Facial. Graduado na FOP - Faculdade de Odontologia de Piracicaba - UNICAMP. Prof. dos cursos de Pós-Graduação em Ortodontia da UNICSUL, Campus São Miguel e Liberdade.

***** Especialista em Saúde Coletiva - São Leopoldo Mandic. Especialista em Saúde Indígena - Universidade Federal de São Paulo. Especialista em Endodontia - Universidade de Ribeirão Preto. Especialista em Gestão em Saúde - Universidade Federal de São Paulo. Mestre em Ciências - Universidade de São Paulo. Professor do curso de Especialização em Saúde Indígena da Universidade Federal de São Paulo. e-mail: pablolemos@gmail.com 
CAMPOS JNS

SANTOS DCL

NEGRETE D

FLAIBAN E

LEMOS PN

OCLUSÃO

DENTÁRIA EM

POPULAÇÕES

INDÍGENAS

\section{8}

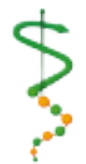

REV. ODONTOL.

UNIV, CID, SÃO

PAULO

$2016 ; 29(1): 57-$

65, JAN-ABR

\section{N T RO DUÇ ÃO}

Estudos realizados a partir de relatórios da OMS e de estudos populacionais de vários países, em 2005, demonstram que as doenças bucais são grandes problemas de saúde pública em todas as regiões do mundo e o maior peso dessas doenças é vivenciado por grupos populacionais desfavorecidos. Os problemas relacionados à oclusão dentária foram destacados, nesses estudos, através do Índice de Estética Dental (DAI), em países da América do Norte e do norte da Europa ${ }^{1}$. O estudo Global Burden of Disease mostrou que, em 2010, aproximadamente 3,9 bilhões de pessoas apresentavam problemas bucais².

Os problemas de oclusão dentária, ou oclusopatias, consistem de anomalias de crescimento e desenvolvimento que afetam músculos e os ossos da maxila e mandíbula no período da infância e da adolescência, podendo produzir alterações funcionais na oclusão, mastigação e fonação, além de alterações estéticas nos dentes e/ou face f $^{\text {. }}$

As causas desses problemas estão relacionadas à hereditariedade e ao meio ambiente, assim como aos estímulos e hábitos presentes durante a formação e desenvolvimento do complexo orofacial. A posição dos dentes nos arcos dentários, o formato e o volume dos ossos maxilares, a forma pela qual se articulam os músculos e articulações envolvidas, modificam-se ao longo do processo de crescimento e desenvolvimento, sob influências do meio ambiente, tratamentos dentários, desgastes, processos patológicos e enveIhecimento. A interação constante entre fatores genéticos e ambientais direcionam o crescimento do complexo crânio-facial, assim como o desenvolvimento de malformações ${ }^{4}$.

Narvai e Frazão ${ }^{5}$ (2008) destacam que as oclusopatias podem gerar transtornos psicossociais com potenciais repercussões na autoestima e no relacionamento familiar e interpessoal dos indivíduos severamente afetados. Além disso, apresentam elevada prevalência tanto na dentição decídua quanto na permanente, dentre os problemas de saúde bucal, ficando atrás apenas da cárie e da doença periodontal ${ }^{6}$. Diante desse contexto, o estudo de suas causas, consequências e prevenção tem se tornado objeto de interesse da saúde pública ${ }^{7}$.

A saúde indígena constitui uma área de práticas da saúde coletiva que busca compreender e intervir nos problemas de saúde dos povos indígenas, contemplando as diferentes concepções do processo saúde-doença e as especificidades étnicas e culturais. Estudos antropológicos apontam para uma menor exigência do sistema mastigatório nas populações de grandes cidades, resultando em dentições com menor função e eficiência mastigatória e um aumento na prevalência e severidade das oclusopatias ${ }^{5}$. Os povos indígenas no Brasil têm passado também nas últimas décadas por uma mudança no modo de viver, na alimentação e no perfil epidemiológico. Assim como em outras populações, a saúde bucal dos povos indígenas guarda uma estreita relação com determinantes culturais, comportamentais e biológicos ${ }^{8,9}$.

Vulnerabilidades dos povos indígenas, tanto biológicas como sociais, como o modo de viver, o território, as relações de contato e o perfil epidemiológico, subsidiaram a proposta da criação e organização do Subsistema de Atenção à Saúde Indígena, em 1999, por meio da lei 9.836/99, conhecida como Lei Arouca. Em 2011, as Diretrizes do Componente Indígena da Política Nacional de Saúde Bucal (PNSB) definem a reorganização do modelo de atenção em saúde bucal direcionada aos povos indígenas, planejadas a partir de um diagnóstico das condições de saúde-doença, subsidiadas pela epidemiologia e informações sobre o território indígena, além de acompanhar o impacto das ações de saúde bucal por meio de indicadores adequados ${ }^{10}$.

As diretrizes da PNSB apontam para a ampliação e qualificação da atenção básica, possibilitando o acesso a todas as faixas etárias e a oferta de mais serviços, assegurando atendimentos nos níveis secundário e terciário de modo a buscar a integralidade da atenção ${ }^{11}$, mas isso ainda não é uma realidade para as populações indígenas. Tanto a atenção primária como a secundária, previstas nos princípios do SUS, e essenciais para a formação de um 
programa de saúde bucal completo, ficaram restritas a um atendimento centralizado, de pouco acesso para os usuários, uma vez que a demanda é enorme e a cobertura muito pequena ${ }^{12}$.

Os estudos científicos publicados envolvem principalmente povos da região amazônica do Brasil $8,13,14,15,16,17,18,19,20,21,22$, $23,24,25,26,27$ e poucos estudos nas regiões Sul-Sudeste 9,28,29,30,31,32,33 e Nordeste $^{34,35,36}$.

Os estudos sobre as condições de saúde bucal dos povos indígenas se concentram em doenças como a cárie dentária e a doença periodontal, de maneira geral com índices altos, atribuídos ao impacto das mudanças alimentares, socioculturais, econômicas, ambientais e à falta de programas preventivos ${ }^{15,16,17,18}$. Há poucos relatos de estudos sobre oclusopatias em indígenas, mas isso tem instigado pesquisadores desde 1964. Neel et al. ${ }^{13}$ (1964) e Pereira e Evans $^{37}$ (1975) realizaram os primeiros estudos no Brasil sobre oclusopatias em povos indígenas. A diversidade sociocultural e a complexidade dos distintos contextos de atenção à saúde bucal dos povos indígenas sugerem a busca por mais informações sobre essas diferentes realidades. A revisão de artigos científicos é um recurso favorável para ampliar esses conhecimentos, considerando a análise de dados epidemiológicos e os aspectos relacionados às oclusopatias. O objetivo deste estudo foi analisar a produção científica sobre oclusopatias em povos indígenas no Brasil no período de 1964 a 2015, verificando os principais problemas de oclusão dentária e os indicadores utilizados.
MÉTODOS

Foi realizada a busca na literatura de artigos que relatassem aspectos sobre oclusão dentária de povos indígenas no Brasil no período de 1964 a 2015. A estratégia utilizada foi a busca eletrônica, utilizando as bases de dados LILACS (Literatura Latino Americana e do Caribe em Ciências da Saúde), PubMed/MedLine (Medical Literature Analysis and Retrieval System Online) e SciELO (Scientific Eletronic Library Online).

Para a identificação dos estudos desta revisão foram utilizados os descritores: oral heath; dental occlusion e dentistry. Esses descritores foram combinados com Indians; Indigenous population; South American Indians. Não houve restrição de idioma. Dos artigos selecionados para a revisão, foram sistematizados e analisados de forma descritiva os seguintes dados: autores, ano de publicação, grupos étnicos, região do país, ano do estudo, tipo de estudo, idioma, temática e indicadores utilizados.

\section{RESULTADOS E DISCUSSÃO}

A busca inicial nas bases de dados identificou 42 estudos. Após a revisão dos títulos, resumos e palavras-chaves foram selecionados nove estudos que abordaram oclusão dentária em populações indígenas no Brasil. Após a leitura dos artigos e suas referências, foram incluídos mais dois artigos relevantes sobre o tema, totalizando 11 artigos para a análise (Tabela 1).

Entre os artigos selecionados, o estudo

Tabela 1. Artigos publicados sobre saúde bucal de povos indígenas no Brasil no período de 1964 a 2015, segundo autores, ano de publicação, etnias, região do país, ano do estudo, tipo de estudo, assuntos abordados pela pesquisa e indicadores utilizados.

\begin{tabular}{|c|c|c|c|c|c|c|}
\hline $\begin{array}{l}\text { Autores/ } \\
\text { Ano da } \\
\text { Publicação }\end{array}$ & $\begin{array}{l}\text { Etnias/Região } \\
\text { do País }\end{array}$ & $\begin{array}{l}\text { Ano } \\
\text { do } \\
\text { estudo }\end{array}$ & $\begin{array}{l}\text { Tipo de } \\
\text { Estudo }\end{array}$ & Idioma & $\begin{array}{l}\text { Assuntos } \\
\text { abordados pela } \\
\text { pesquisa }\end{array}$ & $\begin{array}{l}\text { Indicadores } \\
\text { utilizados }\end{array}$ \\
\hline $\begin{array}{l}\text { Neel et al. }{ }^{13} \\
(1964)\end{array}$ & $\begin{array}{l}\text { Xavante / } \\
\text { Mato Grosso }\end{array}$ & 1962 & Transversal & Inglês & $\begin{array}{l}\text { Cárie dentária } \\
\text { e oclusão }\end{array}$ & $\begin{array}{l}\text { CPO-D e } \\
\text { classificação } \\
\text { de Angle }\end{array}$ \\
\hline $\begin{array}{l}\text { Niswander }{ }^{38} \\
(1967)\end{array}$ & $\begin{array}{l}\text { Xavante e } \\
\text { Baikari (Mato } \\
\text { Grosso) }\end{array}$ & $\ldots$ & Transversal & Inglês & $\begin{array}{l}\text { Cárie dentária, } \\
\text { doença } \\
\text { periodontal, } \\
\text { oclusão, } \\
\text { morfologia } \\
\text { dental, higiene } \\
\text { oral }\end{array}$ & $\begin{array}{l}\text { CPO-D, } \\
\text { IP (Russel), } \\
\text { IHOS, } \\
\text { classificação } \\
\text { de Angle }\end{array}$ \\
\hline
\end{tabular}

CAMPOS JNS SANTOS DCL NEGRETE D FLAIBAN E LEMOS PN

OCLUSÃO DENTÁRIA EM POPULAÇÕES INDÍGENAS

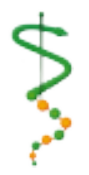

REV, ODONTOL. UnIV. CID. São PAULO

$2016 ; 29(1): 57-$

65, JAN-ABR 


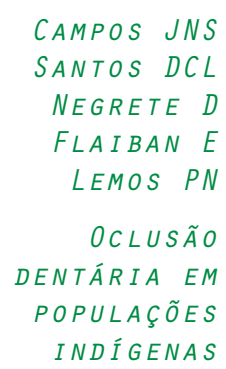

.

$$
\begin{aligned}
& \text { Auto } \\
& \text { Ano } \\
& \text { Pere } \\
& \text { al. }
\end{aligned}
$$

$\begin{array}{lllll}\begin{array}{l}\text { Autores/ } \\ \text { Ano da } \\ \text { Publicação }\end{array} & \begin{array}{l}\text { Etnias/Região } \\ \text { do País }\end{array} & \begin{array}{l}\text { Ano } \\ \text { do } \\ \text { estudo }\end{array} & \begin{array}{l}\text { Tipo de } \\ \text { Estudo }\end{array} & \text { Idioma } \\ & & & & \\ \begin{array}{l}\text { Pereira et } \\ \text { al. }{ }^{39}(1972)\end{array} & \text { Yanomami } & \ldots & \text { Transversal } & \text { Inglês }\end{array}$

(a)

$\begin{array}{lllll}\begin{array}{l}\text { Pereira e } \\ \text { Evans }^{37}\end{array} & \text { Yanomami } & \ldots & \text { Transversal } & \text { Inglês } \\ (1975) & & & \end{array}$

$\begin{array}{llll}\text { Arantes et } & \text { Xavante / } & 1997 & \text { Transversal Português } \\ \text { al.1 }{ }^{6}(2001) & \text { Mato Grosso }\end{array}$

$\begin{array}{lll}\text { Biazevic et } & \text { Kaigang / Rio } 2004 & \text { Transversal Inglês } \\ \text { al. } .^{40}(2005) & \text { Grande do Sul }\end{array}$
Godoy et Fulni-ô / al. ${ }^{41}$ (2006) Pernambuco $\quad \cdots \quad$ Transversal Inglês
Ikpeng,
Kayabi,
Trumai, Yudjá,
Kamaiura,

Mesquita et Kisedje

al.4² (2009) e Waurá

- Parque

Indígena do

Xingu / Mato

Grosso

Regalo et

Não identifica

al. ${ }^{43}(2008)$

etnias-Xingu/ 2007 Transversal Inglês

Mato Grosso

Normando Arara- Iriri e

et al. ${ }^{44}$

(2011)

Arara- Laranjal ...

(PA)

Normando Arara- Iriri e

et al. ${ }^{45}$

(2013)

Arara- Laranjal ...

(PA)
Assuntos

abordados pela Indicadores

pesquisa

Cárie dentária, CPO-D,

doença Canadian

periodontal, Index, índice

oclusão, de Pedersen,

abrasão índice de

cervical, Broca,

desgaste periodontal

oclusal index (PDI)

Cárie dentária, CPO-D,

doença

Canadian

periodontal, Index, índice

oclusão, de Pedersen,

abrasão índice de

cervical, Broca,

desgaste periodontal

oclusal index (PDI)

Cárie dentária, CPO-D, doença ceo-d, INTPC, periodontal, classificação oclusão de Angle

Cárie dentária,

doença CPO-D, CEO,

periodontal, $\mathrm{CPI}, \mathrm{DAI}$

oclusão

Oclusão Classificação

Dentária de Angle

\section{Oclusão Índice DAI \\ Dentária}

Força

mastigatória Medida

entre indígenas de força

e não

(dinamômetro)

indígenas

Transversal Inglês

Oclusão

Dentária

Classificação

de Angle

Coeficiente de

Consanguinidade,

Apinhamento

Prevalência de

e Desgaste

Apinhamento

Dentário 
mais antigo foi encontrado em uma população Xavante no Estado do Mato Grosso. Neel et al. ${ }^{13}$ (1964) avaliaram 46 indivíduos maiores de 15 anos e não encontraram más oclusões significantes. Entre os achados, dois indivíduos apresentaram um leve grau de apinhamento nos dentes anteriores, uma mulher de 15 anos apresentou um pré-molar superior esquerdo supranumerário e dois indivíduos apresentaram um terceiro molar muito pequeno.

Outro estudo com as etnias Xavante e Baikari, realizada por Niswander ${ }^{38}$ (1967), avaliou 204 indivíduos da etnia Xavante e 42 indígenas da etnia Baikari, maiores de três anos de idade. A má oclusão foi avaliada utilizando a classificação de Angle, sendo considerada também uma categoria de oclusão "ideal". Nessa categoria, a relação maxilomandibular era normal (Classe I) e os dentes em quase perfeito alinhamento. Essa classificação permitiu ligeira rotação dos incisivos superiores e inferiores e ligeiro apinhamento dos incisivos inferiores. Quando a sobressaliência foi maior do que 4-5 mm e/ou o trespasse era maior que dois terços dos incisivos inferiores, foi considerada má oclusão. Cinquenta e um indígenas Xavantes não foram classificados por falta de dentes ou pela dentição permanente subdesenvolvida. Portanto, os dados apresentados referem-se a apenas 155 indivíduos. Todos os grupos étnicos foram examinados pelo autor usando os mesmos critérios. Quarenta e cinco por cento dos indígenas Bakairi apresentaram alguma má oclusão, enquanto apenas cinco por cento dos indígenas Xavantes foram afetados. Caracteristicamente, os adultos Xavantes exibiram amplas arcadas dentárias, dentes quase perfeitamente alinhados, mordida topo a topo e extenso desgaste dental (nos indivíduos de 18 a 20 anos de idade os dentes estavam severamente desgastados, apresentando superfícies de mastigação planas).

Pereira e Evans $^{37}$ (1975), em estudo sobre a oclusão e atrição nos Yanomamis, verificaram alta prevalência de má oclusão (71\%) e intensa atrição dentária, concluindo que a dieta dos Yanomamis ocasionava forte atrição dentária com mudanças no plano oclusal, levando a uma relação anterior topo a topo e a uma relação Classe III nos indivíduos de idade mais avançada. Identificaram, também, dois grupos de acordo com os esforços mastigatórios: um primeiro grupo que vivia em área de caça abundante e que tinha abrasão mais intensa e menor quantidade de placa dentária; e um segundo, localizado em região onde a caça era mais escassa e na sua alimentação predominava o consumo de bananas e de alguns tipos de pássaros, com menor abrasão e maior quantidade de placa dentária.

Arantes et al. ${ }^{16}$ (2001) avaliaram a classificação de Angle e presença de má oclusão em 228 indígenas da etnia Xavante da aldeia Pimentel Barbosa no Estado do Mato Grosso. Segundo os autores, a análise foi dificultada pela grande perda dos primeiros molares, sendo que em $32 \%$ dos homens e $45 \%$ das mulheres não foi possível estabelecer o tipo de relação. Nos casos em que foi possível determinar a relação de molares, observou-se uma maioria de $84,3 \%$ de Classe I, uma pequena parte $(12,9 \%)$ de Classe III e uma minoria $(2,8 \%)$ de Classe II. Em relação à má oclusão, a condição " 0 ", que indica ausência de má oclusão, foi observada em 84,6\% da amostra, enquanto apenas $1,3 \%$ apresentaram problema moderado ou severo de oclusão (condição " 2 "). As faixas etárias a partir de 30 anos não apresentaram casos de má oclusão moderada ou severa. Entretanto, os autores consideraram que o diagnóstico de oclusão foi influenciado pelas perdas dentárias que ocorrem com maior frequência a partir dessa idade.

Em 2005, Biazevic et al. ${ }^{40}$ realizaram um inquérito sobre as condições de saúde bucal de 117 crianças de 6 a 12 anos da etnia Kaigang, residentes na reserva de Cacique Doble no Estado do Rio Grande do Sul. Foram utilizados critérios para levantamentos epidemiológicos preconizados pela OMS (índice DAI) e foi observado que $53 \%$ das crianças não apresentaram má oclusão, 39,3\% apresentaram má oclusão leve e apenas 7,7\% apresentaram má oclusão moderada ou grave.

Godoy et al. ${ }^{41}$ (2006) pesquisaram o tipo de oclusão em adolescentes da uma comunidade Fulni-ô no Pernambuco, entre 12 e 15 anos de idade, através de
CAMPOS JNS SANTOS DCL NEGRETE D FLAIBAN E LEMOS PN

OCLUSÃO DENTÁRIA EM POPULAÇÕES INDÍGENAS

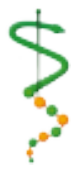

REV, ODONTOL, UnIV. CID, SÃO PAULO

$2016 ; 29(1): 57-$ 65, JAN-ABR 
CAMPOS JNS

SANTOS DCL

NEGRETE D

FLAIBAN E

LEMOS PN

OCLUSÃO

DENTÁRIA EM

POPULAÇÕES

INDÍGENAS

\section{2}

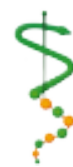

REV. ODONTOL.

UNIV, CID, SÃO

PAULO

$2016 ; 29(1): 57-$

65, JAN-ABR: exame intraoral, classificando em oclusão normal e má oclusão: Classe I, II e III (classificação de Angle). Dados relacionados à má oclusão foram encontrados em $81 \%$ da amostra, sendo que a maioria $(43,6 \%)$ foi classificada como má oclusão de Classe I. A maior prevalência de oclusão normal foi encontrada no gênero feminino, enquanto a prevalência de má oclusão de Classe II e III foi mais alta no gênero masculino.

Um estudo sobre anormalidades dentárias na população indígena do Médio e Baixo Xingu, realizado por Mesquita et al. ${ }^{23}$ (2009), examinou 150 crianças de 5 e 12 anos, de ambos os sexos, em 35 aldeias. Foram utilizados o índice de má oclusão e o índice DAI respectivamente para as idades de 5 e 12 anos, de acordo com a metodologia recomendada pela OMS. No Médio e Baixo Xingu, aos 5 anos de idade a prevalência de problemas oclusais moderados ou severos foi de $1 \%$ e aos 12 anos a prevalência da condição oclusal muito severa ou incapacitante foi de $2 \%$. No Brasil, em 2003, essas prevalências foram de respectivamente $14,45 \%$ e $20,76 \%$, segundo dados encontrados no levantamento epidemiológico nacional de saúde bucal - SB Brasil.

Em relação às forças mastigatórias, Regalo et al..$^{43}$ (2008) compararam a força de mordida de indivíduos indígenas e não indígenas. Oitenta e dois brasileiros com idades entre 18 e 28 anos, foram divididos em dois grupos: 41 indivíduos indígenas do Xingu e 41 indivíduos brasileiros brancos, com 28 homens e 13 mulheres em cada grupo. Os resultados revelaram uma tendência da força de mordida máxima ser maior no grupo indígena quando comparado aos indivíduos brancos.

Normando et al. ${ }^{44}$ (2011) estudaram duas aldeias da etnia Arara, Iriri e Laranjal. O povo Arara-Iriri é descendente de um único casal expulso da aldeia maior. Enquanto a aldeia Laranjal se expandiu por relações não consanguíneas ou raras relações incestuosas, a expansão inicial do grupo Arara-Iriri ocorreu pelo cruzamento de parentes muito próximos, incluindo pais e filhos e entre irmãos, e mais tarde por casamentos entre parentes um pouco mais distantes. A oclusão dentária de
176 indivíduos das duas aldeias foi avaliada. Apesar da semelhança em relação ao desgaste dentário, foi encontrada uma diferença marcante nos padrões oclusais entre as duas aldeias Arara. Na aldeia original, a má oclusão dentária estava presente em cerca de um terço da população (33,8\%); enquanto na aldeia resultante, a ocorrência foi de $63 \%$. Além disso, as características morfológicas da má oclusão foram bastante diferentes entre os grupos: dentre os tipos de má oclusão, as prevalências de Classe I, II e III para os Arara-Laranjal foram de $17,7 \%, 10,8 \%$ e $5,4 \%$ respectivamente. Já para os Arara-Iriri, as prevalências de Classe I, II e III foram de $8,7 \%, 21,7 \%$ e $32,6 \%$ respectivamente. Segundo os autores, os achados sugerem que a genética desempenha um importante papel na etiologia da má oclusão.

O estudo de Normando et al. ${ }^{45}$ (2013) com as comunidades Arara citadas anteriormente, teve como objetivo avaliar o papel da genética e do desgaste dentário na etiologia do apinhamento dentário. Foram examinados 117 pacientes, avaliando-se a presença de apinhamento e desgaste dentário, sendo que após a avaliação clínica, 55 indivíduos sem perda de dentes foram selecionados para medições biométricas através de modelo de estudo. Um alto coeficiente de consanguinidade foi confirmado na aldeia resultante (Iriri), o desgaste dentário não foi significativamente diferente, enquanto uma prevalência significativamente maior de apinhamento dentário foi confirmada na aldeia original (Laranjal). Não foi observada diferença para o tamanho do dente, mas maiores dimensões do arco dentário explicaram um menor nível de apinhamento dentário na aldeia resultante.

\section{CONCLUSÕES}

O aumento de publicações sobre a saúde bucal dos povos indígenas nos últimos anos e o esforço de pesquisadores na busca por determinantes para os problemas de saúde bucal foram perceptíveis. Os estudos começaram a explorar necessidades e problemas de saúde bucal, minimamente investigados anteriormente, como as oclusopatias. Entretanto, o número de publicações ainda é pequeno para buscar 
uma avaliação geral sobre oclusopatias em povos indígenas.

Os diferentes indicadores de oclusão dentária utilizados nos estudos demonstram a necessidade de uma padronização para avaliação dos problemas relacionados à oclusão dentária. Alguns estudos seguem as recomendações da OMS, podendo essa padronização ser um facilitador para a comparação entre diferentes grupos étnicos. Os indicadores mais utilizados foram o índice DAI e a classificação de Angle.

A utilização de metodologias distintas dificulta a comparação entre a prevalência dos vários tipos de má oclusão nas diferentes regiões e grupos étnicos. Entretanto, nos estudos encontrados observa-se a diversidade de padrões relacionados à oclusão dentária. A maioria dos estudos foi realizada em regiões amazônicas e, apesar de apresentar uma caracterização territorial similar, observam-se estudos com alta e baixa prevalência de má oclu- são. A grande mudança no estilo de vida e na alimentação que os povos indígenas vêm enfrentando nos últimos anos pode influenciar diretamente nos problemas relacionados à oclusão dentária e os estudos encontrados evidenciam o caráter multifatorial na etiologia dos diferentes tipos de má oclusão, inclusive relacionado às características genéticas e aos hábitos alimentares.

Conclui-se que mais estudos são necessários para conhecer o perfil epidemiológico das populações indígenas no Brasil no que se refere às oclusopatias e deve-se buscar mecanismos para que os estudos utilizem uma mesma metodologia, de forma a facilitar a comparação entre dados de diferentes estudos. Essas pesquisas podem auxiliar na busca pela integralidade da atenção à saúde indígena, evidenciando os problemas existentes na procura de estratégias de enfrentamento sensíveis às especificidades locais de cada comunidade indígena.

\section{REFERÊNCIAS}

CAMPOS JNS SANTOS DCL NEGRETE D

FLAIBAN E

LEMOS PN

OCLUSÃO

DENTÁRIA EM

POPULAÇÕES

INDÍGENAS
1. Petersen PE, Bourgeois D, Ogawa $H$, Estupinan-Day S, Ndiaye C. The global burden of oral diseases and risks to oral health. Bulletin of the World Health Organization 2005 Sep;83(9):661-9.

2. Marcenes W, Kassebaum NJ, Bernabe E, Flaxman A, Naghavi M, Lopez $A$, et al. Global burden of oral conditions in 1990-2010: a systematic analysis. Journal of dental research 2013 Jul;92(7):592-7.

3. Simões WA. Prevenção das oclusopatias. Ortodontia 1978 11(1):117-25.

4. Planas P. Rehabilitación Neuro-Oclusal (RNO). Barcelona: Salvat; 1987.

5. Narvai PC, Frazão P. Saúde bucal no Brasil: muito além do céu da boca. Rio de Janeiro: Fiocruz; 2008.
6. Thilander B, Pena L, Infante C, Parada SS, De Mayorga C. Prevalence of malocclusion and orthodontic treatment need in children and adolescents in Bogota, Colombia. An epidemiological study related to different stages of dental development. European journal of orthodontics 2001 Apr;23(2):153-67.

7. Lima KC, Paiva ACP, Simplício H. As oclusopatias. In: Ferreira, MaF, Roncalli, AG, Lima, KC. Saúde Bucal Coletiva. Natal: UFRN; 2004. p. 304.

8. Arantes R. Saúde bucal dos povos indígenas no Brasil: panorama atual e perspectivas. In: Coimbra Jr, CEA, Santos, RV, Escobar, AL. Epidemiologia e saúde dos povos indígenas no Brasil. Rio de Janeiro: Abrasco/ Fiocruz; 2003. p. 49.

9. Alves Filho P, Santos RV, Vettore MV. Saúde bucal dos índios Guaraní no Estado do Rio de Janeiro, Brasil. Cad Saúde Pública 2009 25(1):37-46.

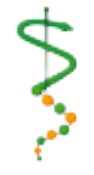

REV, ODONTOL. UNIV, CID, SÃo PAULO

$2016 ; 29(1): 57$ 65, JAN-ABR 
CAMPOS JNS

SANTOS DCL

NEGRETE D :

FLAIBAN E :

LEMOS PN

OCLUSÃO

DENTÁRIA EM

POPULAÇÕES

INDÍGENAS

64

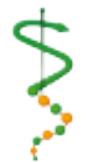

REV. ODONTOL.

UNIV, CID, SÃO

PAULO

$2016 ; 29(1): 57$

65 , JAN-ABR:
I SSN 1983-5183

10. Brasil. Ministério Da Saúde. Secretaria Especial De Saúde Indígena. Diretrizes do componente indígena da política nacional de saúde bucal. Brasília: Ministério da Saúde; 2011.

11. Brasil. Ministério Da Saúde. A política nacional de saúde bucal: registro de uma conquista histórica. Brasília: Ministério da Saúde; 2006.

12. Vargas AMD, Paixão HH. Perda dentária e seu significado na qualidade de vida de adultos usuários de serviço público de saúde bucal do Centro de Saúde Boa Vista, em Belo Horizonte. Ciênc saúde coletiva 2005 dez.;10(4):101524.

13. Neel JV, Salzano FM, Junqueira PC, Keiter F, Maybury-Lewis D. Studies on the Xavante Indians of the Brazilian Mato Grosso. American journal of human genetics 1964 Mar;16(52-140.

14. Tumang AJ, Piedade EF. Cárie dental, doenças periodontais e higiene oral em indígenas brasileiros. Bol Oficina Sanit Panam 1968 64(1):103-9.

15. Detogni A. De volta às origens. Rev Assoc Brasil Odontol 1994 2(1):138-48.

16. Arantes R, Santos RV, Coimbra Jr. CEA. Saúde bucal na população indígena Xavánte de Pimentel Barbosa, Mato Grosso, Brasil. Cad Saúde Pública 2001 17(2):375-84.

17. Rigonatto DD, Antunes JL, Frazao P. Dental caries experience in Indians of the Upper Xingu, Brazil. Revista do Instituto de Medicina Tropical de Sao Paulo 2001 Mar-Apr;43(2):93-8.

18. Parizotto SPCOL. Prevalência de cárie dentária na dentição decídua de crianças da comunidade indígena Kaiowá-Guarani de Mato Grosso do Sul e associação com fatores de risco [Tese]. São Paulo: Faculdade de Odontologia da Universidade de São Paulo; 2004.

19. Pacagnella RC. Perfil epidemiológico de saúde bucal da população do arque indígena do Xingu, entre os anos de 2001 e 2006 [Dissertação]. Ribeirão Preto: FMRP/USP; 2007.
20. Carneiro MCG, Santos RV, Garnelo L, Rebelo MAB, Coimbra Jr. CEA. Cárie dentária e necessidade de tratamento odontológico entre os índios Baniwa do Alto Rio Negro, Amazonas. Ciênc saúde coletiva 2008 dez.;13(6):198592.

21. Arantes R, Santos RV, Frazao P, Coimbra CE, Jr. Caries, gender and socio-economic change in the Xavante Indians from Central Brazil. Annals of human biology 2009 Mar-Apr;36(2):162-75.

22. Lemos PN, Hirooka LB, Nunes SAC, Arantes R, Mestriner SF, Mestriner Júnior W. O modelo de atenção a saúde bucal no Médio e Baixo Xingu: parcerias, processos e perspectivas. Ciênc saúde coletiva 2010 jun.;15(( Suppl 1 )):1449-56.

23. Mesquita LP, Lemos PN, Hirooka LB, Nunes SAC, Mestriner SF, Mestriner Júnior W. Anormalidades dentárias na população indígena do Médio e Baixo Xingu: análise descritiva e comparativa. Reunião Em Pesquisa Em Saúde Coletiva, editor. Faculdade de Odontologia de Piracicaba UNICAMP; 2009.

24. Arantes R, Santos RV, Frazao P. Oral health in transition: the case of Indigenous peoples from Brazil. International dental journal 2010 Jun;60(3 Suppl 2):235-40.

25. Vieira EMM, Ciesielski FIN, Gaetti-Jar$\operatorname{dim}$ EC, Hespanhol D, Lima De Castro EVF, Lima De Castro A, et al. Evaluation of Oral Health in a Community of Native Brazilians of the Umutina Reservation, Mato Grosso State. Int J Odontostomat 2011 abr.;5(1):59-63.

26. Nunes SAC, Lauris JRP, Tomita NE. The implantation process of oral health services in indian communities of the rivers Tiquié and Uaupés- Special Indian Sanitary District for the Alto Rio Negro - Amazonas. Rev Tempus Actas Saúde Col 2013 7(1):183-93.

27. Hirooka LB, Mestriner- Junior W, Mestriner SF, Nunes SAC, Lemos PN, FranCO LJ. Dental caries in mother-child pairs from Xingu. Braz J Oral Sci 2014 mar.;13(1):43-6. 
28. Fratucci MVB. Alguns aspectos das condições de saúde bucal de uma população indígena Guarani Mbyá no Município de São Paulo [Dissertação]. São Paulo: Faculdade de Saúde Pública/USP; 2000.

29. Moura PG. População indígena: condição bucal e estado nutricional materno infantil. Florianópolis: Universidade Federal de Santa Catarina; 2007.

30. Diab AD, Lucas SD. Cárie dentária em crianças indígenas Xakriabá. Epidemiol Serv Saúde 2008 jun;17(2):149-53.

31. Dumont AFS, Salla JT, Vilela MBL, Morais $\mathrm{PC}$, Lucas $\mathrm{SD}$. Índice de necessidade de tratamento odontológico: o caso dos índios Xakriabá. Ciênc saúde coletiva 2008 jun.;13(3):1017-22.

32. Alencar CO, Cortelli JR, Carvalho RB, Miclos PV. Fluorose dentária e condição periodontal de crianças indígenas do município de Aracruz, ES. Periodontia 2011 21(4):80-5.

33. Ulhôa Netto E, Ferreira TFL, Drummond MM, Sanchez HF. Tooth loss and need of denture in Pataxó Natives. RGO, Rev gaúch odontol 2012 jun.;60(2):195-201.

34. Nascimento S, Scabar LF. Levantamento epidemiológico de cárie, utilizando os índices CPO-D, ceo-d e IHOS, nos índios da aldeia Wakri no Estado do Pará. J Health Sci Inst 2008 abr.-jun. ;26(2):246-54.

35. Sampaio FC, Freitas CH, Cabral MB, Machado AT. Dental caries and treatment needs among indigenous people of the Potiguara Indian reservation in Brazil. Revista panamericana de salud publica = Pan American journal of public health 2010 Apr;27(4):246-51.

36. Mauricio HA, Moreira RS. Oral health status of the ethnic group Xukuru from Ororubá: multilevel analysis. Rev bras epidemiol 2014 set.;17(3):787-800.

37. Pereira CB, Evans H. Occlusion and attrition of the primitive Yanomami Indians of Brazil. Dental clinics of North America 1975 Jul;19(3):485-98.
38. Niswander JD. Further studies on the Xavante Indians. VII. The oral status of the Xavantes of Simoes Lopes. American journal of human genetics 1967 Jul;19(4):543-53.

39. Pereira CB, Riesinger A, Riesinger AS, Mooney JB. [Periodontal health, occlusion, wasting and other dental characteristics of the Yanomamis Indians (Brazilian aborigenes). Advanced campus of the Federal University of Santa Maria (Rio Grande do Sul). Rondon project]. Ortodontia 1972 Jan-Aug;5(1):39-54.

40. Biazevic M, Fantin A, Michel-Crosato E. Oral health conditions survey among 6-to-12-year-old children living in the indigenous reserve of Cacique Doble, Rio Grande do Sul, Brazil. Caries Res 2005 39(4):287-340.

41. Godoy F, Rosenblatt A, Guimarães CD. The type of occlusion in adolescents of the fulni-ô Indian community, Pernambuco - Brazil. Odontol clín-cient 2006 out-dez;5(4):307-11.

42. Mesquita LP, Lemos PN, Hirooka LB, Nunes SAC, Mestriner SF, Taba Junior $M$, et al. Periodontal status of an indigenous population at the Xingu Reserve. Braz J Oral Sci 2015 9(1):5.

43. Regalo SC, Santos CM, Vitti M, Regalo CA, De Vasconcelos PB, Mestriner W, Jr., et al. Evaluation of molar and incisor bite force in indigenous compared with white population in Brazil. Archives of oral biology 2008 Mar;53(3):282-6.

44. Normando D, Faber J, Guerreiro JF, Quintao CC. Dental occlusion in a split Amazon indigenous population: genetics prevails over environment. PloS one 2011 6(12):e28387.

45. Normando D, Almeida MA, Quintao CC. Dental crowding: the role of genetics and tooth wear. The Angle orthodontist 2013 Jan;83(1):10-5.

Recebido em 03/10/2016

Aceito em 08/12/2016
CAMPOS JNS SANTOS DCL NEGRETE D

FLAIBAN E LEMOS PN

OCLUSÃO DENTÁRIA EM POPULAÇÕES INDÍGENAS

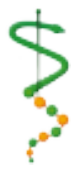

REV, ODONTOL. UNIV, CID, SÃO PAULO

$2016 ; 29(1): 57-$ 65 , JAN-ABR 